\title{
Workshop Report: In Quest of More Affordable and More Accessible Medicines
}

\author{
Isadore Kanfer \\ Rhodes University, Grahamstown, South Africa
}

The 3rd Biennial International Bioequivalence, Biowaivers, and Dissolution Testing Conference took place on December 2-3, 2013 and was cosponsored by the American Association of Pharmaceutical Scientists (AAPS), the Canadian Society of Pharmaceutical Sciences (CSPS), and Rhodes University. The conference was a follow-up of two previous workshops and conferences held at Rhodes University in 2009 and 2011. Members of the organizing committee were Professor Isadore Kanfer-Chair, Professor Rod Walker, Dr. Mike Skinner, Dr. Murray Ducharme, and Mr. Michael Aeroboe.

\section{DAY 1 DISSOLUTION TESTING}

\section{Session 1}

T he conference was officially opened by the Chairperson, Professor Isadore Kanfer, Emeritus Professor, Faculty of Pharmacy, Rhodes University, and Honorary Professor, KLE University, Belgaum, India.

Dr. Erika Stippler (United States Pharmacopoeia) delivered a presentation entitled Updates on Dissolution/Drug Release Testing in the United States Pharmacopoeia. She outlined relevant sections in the USP and associated updates. Details regarding the applicability of the two-tier dissolution in $<711>$ General Chapter on Dissolution for hard or soft gelatin capsules were explored, and the use of additional enzymes, depending on the $\mathrm{pH}$ of the dissolution, was considered. The compositions of Simulated Gastric Fluid (SGF) and Simulated Intestinal Fluid (SIF) have been revised with respect to enzyme activity and stability. She detailed several USP chapters that have been updated. These chapters include $<724>$ Drug Release, and $<2040>$ Disintegration and Dissolution of Dietary Supplements. USP General Chapter $<1724>$ Semisolid Drug Products-Performance Tests is a new general chapter on semisolid products and provides general information on performance testing of semisolid drug products, including a description of three apparatus. Another new USP General Chapter <1094> Capsules: Dissolution Testing and Related Quality Attributes concerns the special considerations of capsule dosage forms.

The next speaker, Dr. Johannes Krämer (Managing Director, PHAST GmbH, Germany), presented a talk entitled The New USP Chapter <1092> Dissolution Method Development and Validation. He explained that the purpose of this chapter is to provide information on the development and validation of dissolution methods and accompanying analytical procedures including the use of automation. Dr. Krämer presented a table of commonly used surfactants and their respective critical micelle concentrations (CMCs). The use of surfactants and the concentration thereof must be justified, and the choice of dissolution apparatus should be based on the formulation to be tested, the practical aspects of dosage form properties, and performance. He explained that the goal when choosing an appropriate dissolution medium and volume is to achieve sink conditions and that the choice of appropriate dissolution medium is dependent on the drug solubility. Generally, a compendial apparatus should be selected, and any changes or the use of a non-compendial apparatus must be justified. He relayed how the use of representative final compounded dosage forms, samples of predefined content, and the variables to be addressed during robustness testing are now included in the general chapter.

\section{Session 2}

Dr. Stippler provided the next presentation entitled Qualification of Dissolution Apparatus. She began with a discussion on concerns relating to variability of results from dissolution testing. She emphasized that high variability in results can make it difficult to identify trends or effects of formulation changes. Furthermore, high variability caused by the test makes it impossible to describe differences in product quality. Dr. Stippler explained that dissolution results may be considered highly variable if the relative standard deviation (RSD) is $>20 \%$ at time points of $10 \mathrm{~min}$ or less and $>10 \%$ at later time points (test and reference). The source of variability should be investigated, and attempts should be made to reduce the variability of the testing procedure/method whenever possible.

Dr. Stippler then addressed USP General Chapter $<711>$ which describes the qualification of dissolution apparatus. Dr. Stippler informed attendees that the previous Apparatus Suitability Test is now known as the Performance Verification Test (PVT), which involves the use of a standard tablet as the reference. The tablet was qualified by a collaborative trial including FDA, USP, and HPB laboratories and about 30 others. Specifications have been established for each individual new lot, and the USP provides a how-to education course entitled Dissolutuion Theory and Best Practices. She mentioned that an FDA Guidance was issued in January 2010 entitled Use of Mechanical Calibration of Dissolution Apparatus 1 
and 2-Current Good Manufacturing Practice and refers to ASTM Document E-2503-07, which describes the Standard Practice of Basket and Paddle Apparatus including the apparatus setup, maintenance, mechanical calibration, and operation. Dr. Stippler referred to the Dissolution Toolkit, which provides enhanced procedures and control limits for mechanical qualification and includes a guide to generate accurate PVT results and a complete information basis to develop SOPs for the GMP environment. USP Apparatus 3 and associated fluid dynamics were discussed, and it was emphasized that operational parameters of the dissolution apparatus may influence the hydrodynamics.

The next speaker was Mr. Hans-Jürgen Knitter whose presentation was entitled Automation of Dissolution Testing.

Automation leads to an increase of efficiency and is time saving since analyst involvement is reduced, the various working steps continue automatically, and for delayed-release products, automatic $\mathrm{pH}$ changes are feasible. In addition, dissolution profiles can be monitored automatically for $\geq 8 \mathrm{~h}$ including several time points. In general, there is a decrease of variability caused by external influences as a result of an increase in precision and robustness. The sources of error are reduced compared with manual handling (human factor). There are, however, several important validation requirements when using automated equipment. These include consideration of adsorption effects (tubing and pumps), filter qualification (suitability and adsorption), carryover effects (vessel to vessel), and verification of the cleaning process (run to run). These systems can be applied to all kinds of dosage forms such as solid oral, suppository, transdermal patches, and suspensions. Semiautomated systems are generally suitable for all kinds of dosage forms. However, some formulations such as suppositories, which may contain fat or oily ingredients, can lead to contamination of the equipment. Mr. Knitter reemphasized that the main disadvantages are that the analyst has to perform various tasks such as, medium filling, tablet placement, cleaning of the vessels, and avoidance of adsorption effects (injection valve, tubing, and filters). The fully automated RoboDis II system was described.

\section{Session 3}

Dr. Johannes Krämer was the first speaker after the lunch break with a presentation entitled USP Chapter 1088-from the Workbench Perspective. Dr. Krämer explained that the purpose of USP <1088> is to provide an overview for the methodology for characterizing the physicochemical properties of a drug substance as well as its associated drug product. The chapter outlines the in vitro and in vivo testing for decision making in formulation, manufacture, and regulatory activities for the product relating to development, regulatory approval, and marketing. It also provides guidance with the main focus on oral dosage forms and complements USP Chapters
$<1090>$ Bioavailability, Bioequivalence, and Dissolution and <1092> The Dissolution Procedure: Development and Validation.

For IVIVC purposes, dissolution profiles are mandatory. The drug substance is characterized according to the Biopharmaceutics Classification System (BCS), and a biowaiver may be possible if the results comply with the FDA guidance on biowaivers based on the BCS.

Dr. Krämer then discussed the requirements for an in vitro-in vivo correlation. This presumes the existence of a rational relationship between a biological property (e.g., $C_{\max }, A U C$ ) or a parameter derived from drug plasma concentrations produced by a dosage form and a physicochemical property or characteristic of the same dosage form (e.g., dissolution behavior) to result in a quantitative IVIVC that can be used to predict clinical performance. He defined the three levels of IVIVC: Level A, Level B, and Level C. Dr. Krämer referred to USP General Chapter <1088> where one way of generating an IVIVC is exemplified using knowledge of relative oral bioavailability and absorption rate of the active ingredient and dissolution data from different modifications of the product obtained under a variety of operational parameters such as $\mathrm{pH}$, agitation, and ionic strength. The chapter also provides an example using deconvolution of drug plasma concentration-versus-time plots to provide mean absorption profiles. Another example showed an in vitro dissolution curve compared with in vivo dissolution rate (absorption rate curve) where the fraction absorbed is plotted versus fraction released in vitro. Dr. Krämer concluded by emphasizing that the dissolution test may not be discriminating, and dissolution conditions may thus need to be modified.

The next speaker was Dr. Gordon Amidon (Charles Walgreen Jr. Professor of Pharmacy, University of Michigan, United States), whose presentation was entitled BCS Subclassification and Drug Product (In Vivo Biopredictive) Dissolution. Dr. Amidon began by explaining the bioequivalence paradigm for orally administered drugs where similar drug plasma levels are expected to result in similar efficacy. Extension of this logic is that similar in vivo dissolution should result in similar drug plasma levels, and thus similar in vitro dissolution of the product could reflect similar in vivo dissolution. He explained that the modern view of transport bioequivalence considers that if two drug products containing the same drug present the drug to the same absorbing membrane the same way, they will be bioequivalent. A link between dissolution and absorption is necessary to predict bioavailability; hence attempts should be made to establish a bioperformance dissolution. He explained that this would involve the development of in vitro dissolution methodology that is predictive of in vivo dissolution. Such methodology is not intended as a QC methodology or a regulatory methodology, but is a drug product development methodology. Dr. Amidon 
then described a proposed BCS subclassification system for dissolution methodologies and presented a table outlining the different subclassifications, conditions, and acceptance criteria. He described important areas of the gastrointestinal tract such as the stomach, duodenum, jejunum, and ileum and their associated $\mathrm{pH}$ and ionic strength environments. He emphasized the important role played by bicarbonate ions and bicarbonate equilibrium in the intestine. The implications of using a bicarbonate buffer were discussed such as the production of bubbles from resulting carbon dioxide and the fact that the in vivo buffer capacity is low (5-15 mM); hence, matching bicarbonate buffer depends on $\mathrm{p} K_{\mathrm{a}}$ and drug solubility. The physiologic relevance of a bicarbonate buffer was then discussed.

Dr. Amidon reviewed the history of bioavailability/ bioequivalence (BA/BE) from the 1960 s to the present day and mentioned that the parameters $C_{\max }$ and $A U C$ were mainly empirical and regulatory dominated. The current view, however, is that historically, the relative bioavailabilitybased view misses the underlying scientific issues involving the complexities of absorption, distribution, metabolism, elimination (ADME), and in particular, in vivo dissolution. He concluded that since BE testing involves the same drug, once it is absorbed the related pharmacokinetics is the same and emphasized that the science of $B E$ is at the absorption site, which for oral dosage forms is the Gl tract. In light of the foregoing, the question that needs to be answered is, What is the best BE test?

\section{Session 4}

Dr. Erika Stippler (USP) was the last speaker of the day with a presentation entitled Drug Product PerformanceContinuing Equivalence. She described the Regulatory Landscape such as the FDA, EMA, and WHO and their respective $B E$ guidelines and requirements. Dr. Stippler described the BCS and pointed out that the classification is related to the highest strength of the drug product according to the FDA, the highest single dose administered according to the EMA, and the highest dose recommended in the World health Organization's (WHO) List of Essential Medicines or the highest dose strength available on the market. When combined with dissolution, the BCS takes into account three major factors that govern the rate and extent of absorption and provides a regulatory tool for replacing certain BE studies with appropriate in vitro tests. BCS class boundaries were then described where degree of solubility has been defined by the different agencies. According to the FDA, when the highest dose strength is soluble in 250 $\mathrm{mL}$ or less of aqueous media over a pH range of 1-7.5 at $37 \pm 1{ }^{\circ} \mathrm{C}$, it is considered a highly soluble drug substance (HSDS). The EMA states that a HSDS is when the highest single dose administered is soluble in $250 \mathrm{~mL}$ or less of buffer solutions of $\mathrm{pH} 1-6.8$ at $37 \pm 1{ }^{\circ} \mathrm{C}$, whereas the WHO definition is when the highest dose strength is soluble in $250 \mathrm{~mL}$ or less of aqueous media over a $\mathrm{pH}$ range of 1.2-6.8 at $37 \pm 1{ }^{\circ} \mathrm{C}$. Permeability definitions also vary somewhat among FDA, EMA, and WHO; according to the FDA, a drug is considered a highly permeable drug substance (HPDS) when the extent of absorption in humans is $>90 \%$ of an administered dose determined from a mass balance reconciliation, absolute $B A$, or intestinal permeability studies/data. The EMA defines a HPDS as one in which the extent of absorption in humans is $>85 \%$ of an administered dose determined by mass balance and its absolute BA, whereas WHO describes a HPDS as one in which the extent of absorption in humans is $>85 \%$ of an administered dose based on mass balance compared with an intravenous (IV) reference dose, but alternative methods are also accepted. The selection of the reference product was then addressed. In the United States, the RLD is required, whereas the EMA requires the innovator product for which efficacy, safety, and quality have been established to be used as the reference product. Dr. Stippler then referred to the USP Medicines Compendium (MC), which is a free online source of public standards for chemical and biological medicines and their ingredients approved by national regulatory authorities and legally marketed in any country. She explained that the $M C$ provides easy access to public standards for medicines and assistance in identifying substandard, spurious, fake, falsified, or counterfeit medicines. It is a reliable reference for developing new products and standard operating procedures (SOPs). Active pharmaceutical ingredients (APIs) with high solubility over the entire $\mathrm{pH}$ range, formulated as rapidly dissolving solid oral dosage forms, can be considered optimally bioavailable (OBA). There is no need for a reference product to evaluate performance. However, to label the product as bioequivalent, the test results must be compared with an appropriate comparator drug product. Dr. Stippler concluded her presentation with a discussion of some examples and summarized that the resulting data can indicate pharmaceutical equivalence and that the API is a highly soluble drug substance. OBA implies $B E$ and no comparator product or comparison study is required; hence, registration can be effected without a regulatory review based only on pharmacopoeial monographs with periodic inspection to assure conformity and labeling as OBA. For all other drug products, in vivo studies are required.

\section{DAY 2 BIOEQUIVALENCE TESTING- CURRENT REGULATORY ASPECTS (UNITED STATES, CANADA, EUROPE, AND SOUTH AFRICA) \\ Session 5}

Dr. Raimar Löbenberg (Director, Drug Development and Innovation Center, University of Alberta, Canada) presented the first talk, entitled Predicting Food Effects Using Dissolution Testing.

He began his presentation by discussing the effect of various media such as USP buffers, Pepsi-Cola, orange juice, and alcohol-containing beverages (varying percentages) on disintegration of calcium tablets used 
as a mineral supplement. The findings and conclusions indicate that with the exception of $5 \%$ alcohol, all beverages have a significant effect on the disintegration time and those beverages should not be used to replace water when ingesting such medications. The mechanisms for decreasing absorption with food include instability of the drug in gastric acids, $\mathrm{pH}$ effects, increasing first-pass metabolism and clearance, and physical/chemical binding with food components, all of which can result in decreased efficacy. Food can also increase absorption where the mechanisms include inhibition of first-pass effects and physicochemical/physiological effects, resulting in longer gastric residence times and increased bile release. Foodformulation interactions can occur where drug absorption from IR products may differ from MR formulations. With MR dosage forms, dose-dumping can occur in the presence of alcohol. Turning to solubility, the question that needs to be answered is, Which solubility (i.e., in water, in gut juices, formulation-drug solubilization) is the right one? The use of solubility enhancers, such as CoQ10, quercetin, and parthenolide, and conventional (pharmacopoeial) and biorelevant dissolution media (FaSSIF and FeSSIF) were then discussed. The key differences between these media were emphasized. In the case of biorelevant media, both FaSSIF and FeSSIF contain differing amounts of $\mathrm{Na}$ taurocholate and lecithin depending on $\mathrm{pH}$ (6.5 or 5.0), whereas FeSSIF contains $\mathrm{Na}$ taurocholate. The influence of low and high quality $\mathrm{Na}$ taurocholate and egg lecithin on the solubility of glyburide and also the influence of $\mathrm{pH}$ and surfactant (sodium lauryl sulfate) concentration on the solubility of piroxicam were shown. Dr. Löbenberg referred to a very useful resource, the DrugBank data base, which is a unique bioinformatics and cheminformatics resource that combines detailed drug data (chemical, pharmacological, and pharmaceutical) with comprehensive drug target (sequence, structure, and pathway) information (www. drugbank.ca). He also described the application of the flow-through apparatus and GastroPlus software to predict food effects.

The next speaker was Dr. Erika Stippler (USP), whose presentation was entitled USP Flow-Through Apparatus: Applications. She began by describing the history of this apparatus, which was introduced in 1981 in the opensystem configuration and adopted by the Deutscher Arzneimittelcodex. In that same year, the International Pharmaceutical Federation (FIP) proposed the use of the flow-through apparatus for poorly soluble and extendedrelease formulations. It was incorporated into the USP 23NF 18 in 1995, assigned as USP Apparatus 4, and accepted by the Japanese Pharmacopoeia in 1996. Dr. Stippler described the apparatus and its associated operating conditions and specifications relating to the cells, pump, media, and whether an open or closed system is used. The open and the closed systems were described. The flowthrough apparatus can be used for various dosage forms such as:
- Solids: tablets, capsules, implants, powders, granules.

- Semisolids: suppositories, soft gelatin capsules, ointments, creams, etc.

- Liquids: suspensions.

Drug release from semisolid dosage forms was then discussed with particular reference to the USP chapter on dietary supplements $<2040\rangle$. Here the FTC system is suited for lipid-filled gelatin capsules, hard and soft gelatin capsules, and gelatin-coated tablets that do not conform to the dissolution specification where the two-tier dissolution test using enzymes is used. There is also a special powder cell that can be used for apparent or intrinsic dissolution studies (Ph. Eur. 2.9.43). A cell for use with suppositories and soft gelatin capsules is described in Ph. Eur. 2.9.42 for use with lipophilic dosage forms. Elution from drugeluting stents, such as sirolimus stents, can be evaluated using the FTC system. It was emphasized that elution and not dissolution is monitored from coated stents. Another application using the FTC apparatus is the measurement of drug release of liposomes with a dialysis adaptor. USP Apparatus 4 has also been described for ocular implants (Browne, D. C.; Kieselmann, S. Dissolution Technol. 2010, 17 [1], 12-14).

The next speaker was Professor R. B. Walker (Dean of Pharmacy, Rhodes University) who discussed Bioequivalence Requirements for Market Approval of Generic Drug Products in South Africa: Current Guidelines. Professor Walker outlined the basic South African requirements and emphasized that substitution of a branded medicine by an interchangeable multisource medicine is mandatory by law. Since an interchangeable medicine by definition must be therapeutically equivalent to the innovator product that is used as the reference drug product in a BE study, this presents several issues in South Africa relating to the choice of reference product, using dissolution testing as a general biowaiver for $\mathrm{BE}$ and a consideration of pharmaceutical alternatives. The current Biostudies Guideline was updated in 2011 and contains definitions, design and conduct of studies, and $B A / B E$ requirements for oral products, parenteral solutions, topicals, and others. Also included are waivers using in vitro studies and BCS considerations. Professor Walker drew attention to a recent government publication, Gazette Number 37032, Notice 870 of 15, November 2013, where Regulation 2(1) paragraph (a) was amended. This refers to the requirements for therapeutic equivalence, which includes pharmaceutical alternatives. Professor Walker pointed out that acceptance of a pharmaceutical alternative is not in keeping with international requirements for therapeutic equivalence to declare a product interchangeable. Waivers of in vivo BE studies for solid oral dosage forms can be obtained using dissolution profiles as described in the South African Dissolution Guidelines where three media are described and other associated calculations and acceptance criteria. Dissolution studies 
can also be used for proportionately similar formulations; immediate-release tablets, modified-release products such as beaded capsules, and tablets of lower strength may be approved. The BCS can also be applied for a biowaiver. The approach to the choice of a reference product for use in a BE study is listed in the Pharmaceutical and Analytical Guideline of June 2011. Reference products registered in South Africa but procured in another country may be used. As in most other countries, the bioequivalence acceptance criteria involve the use of ratios of $A U C_{\text {test }} / A U C_{\text {ref }}$ and $C_{\max \text { test }} / C_{\max }$ ref and $90 \% \mathrm{Cl}$ of $0.8-1.25$ interval for the former but $0.75-1.33$ for the latter ratio.

\section{Session 6}

Following a tea break, the next talk entitled New Canadian Guidelines for Bioequivalence was presented by Dr. Murray Ducharme (Learn and Confirm Inc., Quebec, Canada). This was essentially an update on Canadian BA/ $B E$ requirements where he presented definitions and regulations and discussed the requirements, which were compared with those of the EMA and the FDA. Whereas most of the $B E / B A$ definitions are the same throughout most countries, he mentioned the term identical medicinal ingredient (IMI), which is a specific Canadian term described in the 2003 policy document and defined as follows: Pharmaceutically equivalent drug products should contain chemically identical, but not necessarily physically identical, medicinal ingredients. The following are considered identical: salts, esters, complexes of the same active moiety, different isomers or mixtures with different proportions. The Canadian regulatory authorities have since 1972 been requesting tougher criteria for complicated drugs such as narrow therapeutic index and drugs exhibiting nonlinear kinetics. They were the first to introduce tougher criteria for critical dose drugs (CDD) and also for early onset of action drug products.

Dr. Ducharme indicated that the original longstanding policy of 1995 relating to the reference product that must be used in a BE study for the market approval of a generic product for Canada still applies. For drugs exhibiting nonlinear pharmacokinetics, Health Canada requires studies on the most discriminative strength plus a fed study. If due to limited solubility, studies should be performed at both low and high strengths. For long halflife drugs, truncated $A U C_{0-72}$ instead of $A U C_{\mathrm{t}}$ and $A U C_{\text {inf }}$ if $t_{1 / 2}>24 \mathrm{~h}$ are required.

Currently, Canadian definitions, regulations, and guidances related to $\mathrm{BE}$ are often different from most others and unique. Some requirements are aligned with those of EMA and others with those of the FDA.

The following presentation entitled Options for the Bioequivalence Assessment of Topical Products was made by Professor Isadore Kanfer (Emeritus Dean \& Professor, Rhodes University).

He contended that the biopharmaceutics and bioequivalence assessment of locally acting products is a much neglected area and the demonstration of $\mathrm{BE}$ for generic topical products as well as for post-approval changes present daunting challenges. He emphasized that new approaches are urgently required. In the case of topical products where the active ingredient is not intended to be absorbed into the systemic circulation, apart from a single exception in the case of topical corticosteroids where the human skin blanching assay (HSBA), also known as the FDA's Vasoconstriction Assay (VCA), is used, clinical end-point studies in patients are required. However, various methods such as dermatopharmacokinetic (tapestripping), drug diffusion/perfusion such as dermal microdialysis (DMD), and dermal open-flow microperfusion (DOFM) are being investigated by researchers. Professor Kanfer mentioned that consideration should be given to the different intentions of a topical product such as those intended for local action on or in the skin and the vicinity below and those intended for regional delivery to deeper tissue such as muscle tissue, as in the case of nonsteroidal anti-inflammatory agents (NSAIDS). A well-established method for the BE assessment of topical corticosteroids, the HBSA (or VCA), was first used in by McKenzie and Stoughton in 1962 and is based on the observation of Hollander et al. in 1950 that following application of a topical corticosteroid, a whitening or blanching of the skin occurs. The method was accepted by the FDA and published as a guidance in 1995. The acceptance criteria for the declaration of $\mathrm{BE}$ requires that only the ratios of $A U E C_{t} / A U E C_{\text {ref }}$ be used where the $90 \% \mathrm{Cl}$ and limits of 0.8-1.25 using untransformed data are considered. A dermatopharmacokinetic method using tape-stripping was investigated, where the amount of drug permeation into the stratum corneum (SC) is determined. The method utilizes adhesive tape strips, is relatively noninvasive, and sequentially removes layers of SC. The FDA issued a draft guidance in 1998 where a tape-stripping procedure was recommended and described. However, the FDA withdrew the guidance on the basis of major concerns regarding the reproducibility of this procedure among laboratories. The latter concern was based on contradictory results generated by two reputable independent laboratories involving the $B E$ assessment of tretinoin gel products. This was due to a number of limitations, in particular the sources of variability and control issues. However, in spite of those shortcomings, researchers have continued to explore the potential of TS for the BE assessment of topical products. Some of the questions raised include the dose duration (i.e., how long the product should be left on the skin prior to undertaking TS). This is a very important consideration since it must be assured that the dose duration will provide the necessary discriminatory power to identify differences or equality between test and reference products. Professor Kanfer emphasized that it is also important to normalize skin thickness between subjects, which can be done using transepidermal water loss (TEWL) measurements. He went on to give several examples of the use of TS for 
the $\mathrm{BE}$ assessment and also described the application of dermal microdialysis using the bioequivalence assessment of a $2.5 \%$ ketoprofen gel as an example. He concluded his talk with a presentation of data using in vitro methods for the assessment of $B E$ in accordance with a recent draft guidance issued by the FDA for BE assessment of acyclovir topical ointment.

\section{Session 7}

The next speaker was Dr. Murray Ducharme, and his presentation was entitled Biosimilars: Regulatory Requirements for Market Approval. Dr. Ducharme began his presentation by comparing the characteristics of biologics versus small molecules. Biologics generally have large molecular weights compared with the low molecular weight small molecules. Biologics have complex physicochemical (tertiary structure, possible glycosylation) properties, whereas the physicochemical properties of small molecules are well defined. Furthermore, the synthesis of biologics is via recombinant DNA compared with chemical synthesis of small molecules, and the former are generally administered parenterally and associated with significant immunogenicity compared with small molecules, which are generally administered orally and whose immunogenicity is rare. The main difference from a PK/PD or clinical pharmacology standpoint between a biologic and a small molecule drug is that the latter can be fully characterized. For example, cyclosporine will be the same whether it is in a reference or test formulation whereas a biologic cannot at this time be fully characterized, hence a reference biologic and a test biologic are not identical although they may be deemed similar. Consequently, no batch of biological product is identical to another batch of the same product but may be similar or biosimilar (Schneider, C. K. Ann. Rheumatic Dis. 2013, 72 (3), 315318). When considering approval requirements for small molecule drug products, their PKs and PDs can be shown to be equivalent, whereas for biologics, because of uncertainty in characterization, similar PK profiles do not necessarily translate into similar PD responses. Conversely, similar PD responses can be achieved despite different PK profiles. Dr. Ducharme presented the following argument: Should the PD measure be valid in terms of similarity of efficacy, safety, or both, then should PD and PK be all that is needed for similarity of efficacy or safety? He then addressed the issues of follow-on biologics/biosimilar submissions that are not yet switchable (i.e., 351 [k] BLA of the PHS Act in the United States, none in Canada, and biosimilar in EU), whereas for new biologics, full new biologic applications are required and include complete safety and efficacy study reports (i.e., 351 [a] of the PHS Act in United States, biologic submission in Canada, MAA in EU). He referred to the FDA update regarding biosimilars (September 2013) where 41 initial meetings were held and 17 INDs submitted for biosimilars. The first approved interchangeable biosimilar will have 12 months of market exclusivity.
Considering the pharmacokinetic metrics, when comparing two different formulations of a small molecule drug product, $C_{\max }, A \cup C_{t}$, and $A U C_{\text {inf }}$ suffice. However, this is not the case with biologics since "identicality" cannot be proved, so these metrics will not suffice as there can be differences in other PK parameters such as the terminal half-life and also the distribution half-life and initial half-life (e.g., anti-HER2).

Considering PD metrics and biomarkers, from a regulatory point of view, only validated biomarkers that have proved their dues can be used as a marker of efficacy to replace a clinical endpoint. However, regarding similarity of biologics and biosimilars, it may not be necessary to be so stringent regarding biomarkers.

Most biologics undergo multiple changes in manufacturing process after the start of commercialization (e.g., Remicade [infliximab] > 35 changes, MabThera [rituximab] $>5$ changes), and most comparability exercises have been minimal for original biologics, especially before the advent of biosimilars.

Dr. Ducharme concluded his presentation by emphasizing that biologics possess characteristics that set them apart from small molecule drugs. Clinical pharmacology principles do, however, apply to biologics as they do for small molecules, and the development of biosimilars is complicated, difficult, and very costly. The metrics for PK studies to meet acceptance are more numerous than for small molecule drugs.

The next speaker was Dr. Barbara Davit (formerly FDA and now Executive Director, Biopharmaceutics, Merck Research Labs, United States). The title of her presentation was Comparison of International Bioequivalence Recommendations.

She referred to the International Generic Drugs Regulators Pilot (IDGRP) from 2011 to 2013, where members of these various organizations met to discuss harmonization and convergence of generic drug development. She listed the jurisdictions and organizations surveyed for comparison of BE requirements; these included Australia, Brazil, Canada, China, European Medicines Agency (EMA), Japan, Mexico, Singapore, South Korea, Switzerland, Taiwan, United States, and WHO. She pointed out that Australia and Switzerland often follow EMA guidelines, and Singapore follows the Association of Southeast Asian Nations (ASEAN) guidelines. Different ways of determining appropriate reference products for BE of generic drug studies exist in various regions of the world.

For example, the United States, Australia, and Japan require pharmaceutical equivalents (i.e., same dosage forms containing the same amounts of drug substance) to be compared in BE studies. In Canada, ASEAN and WHO permit pharmaceutical alternatives where the product is intended for the same route of administration and contains the same amount of the same drug substance; hence, a tablet dosage form can be compared with a capsule dosage form. The EMA, on the other hand, permits products of the 
same dosage but containing a different salt form of the API to be compared. Generally, most regulatory agencies require that the reference be approved and marketed in the jurisdiction in which the generic marketing is intended. Australia, Canada, and Switzerland allow, in limited situations, the use of a reference product that is approved and marketed outside of the country. However, certain rigorous criteria must be met. The WHO Prequalification of Medicines Programme (PQP) defines the reference as the product that is usually the first authorized for market. It must be purchased from a well-regulated market in a country participating in the International Conference on Harmonisation (ICH). However, she emphasized that WHO is not tied to any specific national market.

The FDA recommends both a fasting and a fed study for all immediate-release (IR) products. Most other jurisdictions require a fed study for IR products only under certain conditions, such as in Brazil, if there is a known food interaction. EMA and ASEAN require a fed study if the product labeling states that the drug must be taken with food. Canada requires a fed study if there is a significant safety risk when the drug is taken with food. In the case of modified-release orally administered products, all jurisdictions surveyed require both fasting and fed studies. Canada, EMA, South Korea, and the United States specify a high-fat, high-calorie meal for fed studies; Japan requires a 700 -calorie meal with fat content not $>20 \%$; WHO suggests that the meal be based on local custom and diet, and the guidelines of the other surveyed agencies did not specify meal content. Dr. Davit then considered the situation when multiple dosing may be justified. In Brazil, Japan, and also the WHO, multiple-dose studies are acceptable to reduce within-subject variability. In Taiwan, ASEAN, and the WHO, multiple-dose studies are also provided as an option for drugs that exhibit nonlinear kinetics. China, EMA, South Korea, and Taiwan require multiple-dose studies for modified-release (MR) products, and Canada may consider such studies for MR formulations. All the agencies surveyed indicate when pivotal BE studies must be done in patients. Australia, Canada, EMA, and the United States accept group-sequential designs for BE. Dr. Davit then discussed the various parameters and acceptance criteria used to declare $\mathrm{BE}$. A discussion of the requirements for highly variable drugs (HVDs) and narrow therapeutic index (NTIs) drugs then followed.

Dr. Davit described the various terms used for drug products requiring therapeutic concentration or pharmacodynamics monitoring and when product labeling indicates a narrow therapeutic range. These include critical dose drugs (CDD), narrow therapeutic index (NTI) and narrow therapeutic range drug products. Each agency guideline provides detailed information on such products. She then posed the question, "How are BE acceptance limits set for such products?" In Canada, the limit for $90 \% \mathrm{Cl}$ of AUC is $90.0-112 \%$ and $80-125 \%$ for $C_{\max }$. China, ASEAN, and the WHO guidelines recommend that it may be necessary to tighten BE limits based on clinical justification. The EMA guideline requires $90 \% \mathrm{Cl}$ for $A U C$ of $90.00-111.00 \%$ and also for $C_{\max }$ when this parameter is of particular importance for safety or efficacy. The Japanese guidelines recommend $90 \% \mathrm{Cls}$ of both $A U C$ and $C_{\max }$ falling between $90.00-111.00 \%$, whereas the United States requires that the BE limits for $A U C$ and $C_{\max }$ be scaled using the within-subject variability of the RLD. The assumption is that NTI products have low within-subject variability and thus require narrow acceptance limits (e.g., warfarin and tacrolimus).

Finally, biowaivers were considered, and all the surveyed agencies permit biowaivers for parenteral solutions provided they have the same active and inactive ingredients in the same amounts (Q1/Q2). When considered for other routes of administration, the test and reference products should not differ in excipients that could affect absorption or availability. Also, for products where an additional strength is required for market approval (i.e., one strength previously approved), in vitro dissolution or release testing may be acceptable but the strengths considered for a biowaiver must be proportionately similar to the product strength used in the market approval biostudy.

Application of the Biopharmaceutics Classification System (BCS) for biowaivers was then addressed. Application of the BCS is currently considered by Australia, ASEAN, China, EMA, South Korea, Switzerland, the WHO, and the United States. Canada has also recently accepted the use of BCS for biowaivers. Dr. Davit explained that the various jurisdictions surveyed have some differences in their requirements relating to solubility, $\mathrm{pH}$ range for the solubility/dissolution studies, and acceptance criteria for high in vivo permeability. The types of biowaiver, dissolution requirements, paddle speeds, and $T$ and $R$ formulation requirements were also discussed. The EMA, Australia, and Switzerland, for example, consider biowaivers for Class 1 and 3 drugs whereas the United States and South Korea only permit Class 1 . The WHO recommends biowaivers for Class 1 and 3. Acceptance criteria vary somewhat between jurisdictions, where Rapid is $\geq 85 \%$ in $30 \mathrm{~min}$ and Very Rapid is $\geq 85 \%$ in $15 \mathrm{~min}$. As far as the formulation requirements are concerned, generally the $T$ and $R$ should be qualitatively the same (Q1) and quantitatively the same (Q2), except the EMA, Australia, and Switzerland for Class 1 products that may differ. The same formulation requirements are recommended by the WHO, where differences are accepted for Class 1 products but not for Class 3. Dr. Davit concluded her presentation by noting that there are more similarities than differences among the 13 regulatory agencies surveyed.

Dr. Davit also presented the next talk, entitled Dissolution Evaluation in FDA's Office of Generic Drugs. She began by discussing the objectives of setting discriminating dissolution specifications which include, among others, assuring batch-to-batch quality, providing 
process control and $\mathrm{QA}$, detecting relevant product changes to ensure consistent performance, evaluating post-approval changes, and if possible, predicting in vivo performance to reduce unnecessary human testing. The various steps and components required to develop a predictive dissolution method were then outlined, and the conditions required to be evaluated include the choice of apparatus; stirring speed; and dissolution medium type, volume, composition, and associated $\mathrm{pH}$ and additives such as surfactants that may be required. An important objective using dissolution testing is to develop IVIVCs where three correlations, $A, B$, and $C$ in order of importance and utility, have been recommended. The FDA requires in vitro dosedumping tests in alcohol for some MR products, such as those containing opioids, or if the clinical division asks OGD to perform the test and if the Approval Summary shows that the test supported approval of the RLD. It is considered a safety test supporting therapeutic equivalence where the generic should show rugged performance in alcohol. If the generic releases more rapidly in alcohol, the rate should be comparable to that of the RLD. The in vitro dose-dumping in alcohol method was described by Dr. Davit where the basic medium is $0.1 \mathrm{~N} \mathrm{HCl}$ using the same volume and apparatus as the regulatory method with varying amounts of alcohol beginning with $0 \%$ and then $5 \%, 20 \%$, and $40 \%$ alcohol added with sampling every $15 \mathrm{~min}$ up to $2 \mathrm{~h}$. Twelve units of all the strengths of the test and reference must be tested.

Dr. Davit mentioned that there were fewer than ten ANDAs with IVIVC data submitted to OGD over a 15-year period, and several were not acceptable or were withdrawn for various reasons. Several were currently under review at OGD and one was acceptable where the IVIVC supported change in dissolution specifications. She also mentioned some common product dissolution deficiencies such as $>12$ units used or where not all strengths of the T and RLD were used. If data from a stability batch are used to change dissolution method specs, data from three production batches can be used. Other deficiencies included incomplete dissolution validation reports, proposed specification ranges too wide, dissolution data variability $(\% \mathrm{CV})$ too high , FDA-recommended method not tested, and in the case of DR products, the means to change from acid to buffer.

The next speaker was Dr. John Gordon (WHO) with a talk entitled Bioequivalence and Biowaivers: WHO Update. Dr. Gordon began by describing the United Nations Prequalification Programme (PQP) for Priority Essential Medicines. This was an action plan from 2001 for expanding access to selected priority medicines with the objective of ensuring the quality, efficacy, and safety of medicines procured using international funds (e.g., GFATM, UNITAID). The components include the evaluation of quality, safety, and efficacy of prioritized essential medicines, inspections of manufacturers, and monitoring of the products after their prequalification. Also included are prequalification of quality control laboratories and building capacity of regulators, manufacturers, and quality control laboratories. Dr. Gordon described the primary categories of medicines on the list, which includes products for treatment of HIV/ AIDS, malaria, and tuberculosis. Subsequently, products for reproductive health, influenza, acute diarrhea, and neglected tropical diseases were added. Potentially, other products, where needed, may be added to the list.

Dr. Gordon referred to the WHO guideline for multisource (generic) pharmaceutical products and registration requirements to establish interchangeability (WHO Expert Committee on Specifications for Pharmaceutical Preparations. No. 937, 2006, Annex 7). He pointed out that Annex 7 was currently under revision, Also, guidance on the selection of comparator pharmaceutical products for equivalence assessment of interchangeable multisource products was under revision, and he pointed out that the PQP provides lists of PQP comparator products on their website. There are also additional PQP BE-related guidances on that website. He summarized the WHO requirements for a typical BE study design including the requirements for biowaivers. Turning to pediatric medicines, for many pediatric strengths such as those used for HIV/AIDS, there are no equivalent strength comparator products. $P Q P$, in consultation with experts, is exploring the scientific basis for accepting biowaivers for pediatric strengths of products whose adult strengths are eligible for a BCS-based biowaiver. Biowaivers are only applicable for eligible APIs on the PQP list, and there is an additional solubility criterion to account for smaller fluid volumes in the pediatric stomach (e.g., $50 \mathrm{~mL}$ ). Furthermore, the excipient comparisons should be conducted on a proportional scale, and comparative dissolution studies carried out in media of at least three $\mathrm{pH}$ values with single- and multiple-unit comparisons.

Dr. Gordon discussed the need for a complete and acceptable analytical method validation. All procedures including handling and storage should be validated according to the EMA guidance Guideline on Bioanalytical Method Validation (2011). He also referred to new FDA and Japanese guidelines and noted that incurred sample reanalysis (ISR) is expected. Dr. Gordon then turned to the issue of the source of comparator products, which should be obtained from well-regulated regulatory authorities with stringent requirements (i.e., ICH participants, which include EU, Japan, and the United States and ICH observers such as Canada and EFTA as represented by Switzerland). Other countries associated with $\mathrm{ICH}$ through legally binding mutual recognition agreements include Australia, Norway, Iceland, and Liechtenstein. He mentioned that if a WHO comparator product cannot be located from $\mathrm{ICH}$-associated markets, the PQP should be consulted for assistance in identifying pharmaceutical distributors and identification of alternate markets for sourcing particular products. He also mentioned that there are instances when a comparator is not available in the $\mathrm{ICH}$ region, such as trazodone $300 \mathrm{mg}$ (Terivaldin $250 \mathrm{mg}$ Sanofi-Aventis, South Africa only) and 
artesunate/amodiaquine 100/270 mg FDC (CoarsucamSanofi-Aventis). He informed the meeting that the PQP is constantly evolving and that the website should be visited regularly for updates on guidances including advice on the design of BE studies for specific APIs, application forms, and comparator information.

The next presentation entitled, BCSs and Beyond: Limitations and Harmonization, was by Dr. Gordon Amidon. He began his talk by drawing attention to the meaning of terms such as drug and drug product. He said that this ambiguity reaches back as far as Section 6 of the Pure Food and Drug Act of 1906, which defines drug as "any substance intended to be used for the cure, mitigation, or prevention of disease of either man or other animals." Dr. Amidon emphasized that the product label is very important since the product must do what the label states. Hence, how do we ensure the labeling? Historically, bioequivalence was the beginning of the development of regulatory standards in 1974, where a report entitled Drug Bioequivalence was provided by the Office of Technology Assessment Drug Bioequivalence Study Panel. In that report, the First Principle (Central Dogma) of Bioequivalence for oral drugs, systemically active was described. Dr. Amidon emphasized that the report indicated that similar plasma drug concentrations between products relate to similar pharmacodynamics (safety and efficacy) and that similar in vitro dissolution should relate to similar plasma drug concentrations, implying that in vitro dissolution should relate to in vivo dissolution. Subsequently, however, from 1960 until the present time, the concepts of BABE are mainly empirical and regulatory dominated where metrics such as $C_{\max }$ and $A U C$ are used. Little in the way of biopharmaceutical mechanisms is reflected-absorption, distribution, metabolism, and elimination (ADME) are very complex processes and cannot simply be dealt with empirically. BA and BE have been governed by regulatory standards from about the 1970s. Considering the current situation regarding $\mathrm{BABE}, \mathrm{Dr}$. Amidon contended that historically, it is a relative bioavailability-based view that misses underlying scientific issues such as in vivo dissolution.

Dr. Amidon then turned to the issue of biowaivers where he emphasized that a waiver of in vivo BE studies is not a waiver of $\mathrm{BE}$. He emphasized that for $\mathrm{BE}$, it is important to relate the plasma paradigm to the actual mechanism (oral). In other words, how does a plasma drug concentration-versus-time profile relate to movement of drug through the GIT? A consideration of human jejunal permeability (BCS) is critical and may be considered as the Gold Standard.

The final presentation entitled Recent Changes in the Organization and Process Implementation at the U.S. FDA Office of Generic Drugs was delivered by Lane Christensen (Office of Generic Drugs [OGD], CDER/FDA). He began by defining various terms used by the FDA such as GDUFA (Generic Drug User Fee Amendment) and discussed the objectives of this program and also those of the OGD. The implementation of GDUFA is intended to make changes to the generic drug program at FDA, the generic drug review in OGD and communication with industry, and inspections. The effect on industry is to improve the quality of applications and affect the number of review cycles and communication with the FDA. Dr. Christensen explained that GDUFA involves process identification, mapping, and process improvement. It also includes the development of strategy to reach goals/metrics, implementation, and operational aspects. He drew attention to the GDUFA goals and commitments, which are published on the GDUFA website (http://www.fda.gov/gdufa), and the GDUFA Commitment Letter (http://www.fda.gov/downloads/ ForIndustry/UserFees/GenericDrugUserFees/UCM282505. pdf). This was followed by a review of GDUFA performance goals for the period 2013-2017 relating to ANDAs, amendments, backlogs, and correspondences as well as hiring, procedural, and inspection performance goals.

Several new guidances for industry were published: BE for Metered-Dose Inhalers (MDIs), BE for Ophthalmic Emulsions, and BE for dry-powder inhalers (DPIs). Public meetings and comments are scheduled for 2014. Priorities for regulatory science projects include Postmarket Evaluation of Generic Drugs, Equivalence of Complex Products, Equivalence of Locally Acting Products, Therapeutic Equivalence Evaluation and Standards, and Computational and Analytical Tools. Dr. Christensen mentioned that OGD is the interface for ANDA applicants to interact with the Generic Drug Program and includes all of CDER and other FDA units as well as the Office of the Commissioner, Center for Biologics Evaluation and Research (CBER), Center for Devices and Radiological Health (CDRH), and the Office of Regulatory Affairs (ORA). For GDUFA years one and two, industry has received a free pass, being able to submit DMFs with the ANDA. The complete response letters require adequate responses to address all deficiencies received in the CR. Partial responses will not be accepted and will thus not be processed as a resubmission and will not start a new review cycle. A timely (within one year) response is necessary-21 CFR 314.65 can be enforced and the application withdrawn administratively to prevent abuse and wasting of GDUFA resources. For easily correctable deficiencies (ECDs), a timely response is necessary (within 10 U.S. business days), which will allow OGD reviewers to finish their reviews. However, it is still possible to get major/minor deficiencies in the CR. The apparent industry motto of "file first, develop later" will no longer be tolerated. On February 22, 2013, FDA Commissioner Margaret A. Hamburg stated, "The FDA's administration's Center for Drug Evaluation and research is working on creating a new office of pharmaceutical quality." Dr. Christensen enunciated the organizational principles of the new quality office such as risk-based resource management and decision making, maximal development and utilization of staff expertise, proactive oversight of 
product quality and manufacturing facilities through surveillance and integration of review and inspectional functions. The new office should provide seamless review and surveillance from evaluation and verification of design to surveillance of production and product quality including supply chain and quality management system. The operational aspects of the new quality office were then discussed. It is the intention to establish clear standards for review and inspection and to have clear enforcement policies where the same standards will be applied for all drugs-lifecycle approach. Specialization and team review will also be addressed to result in integration of review and inspection for a quality assessment with necessary clinically relevant standards and surveillance using quantitative metrics. The current product quality review and monitoring model, which involves specification and process evaluation and the associated CGMP inspection, was compared with the new model where product design and standard evaluation will run in parallel with the process design and facility evaluation and associated surveillance and CGMP inspection.

Dr. Christensen concluded his presentation with an outline of the organizational and process implementation.

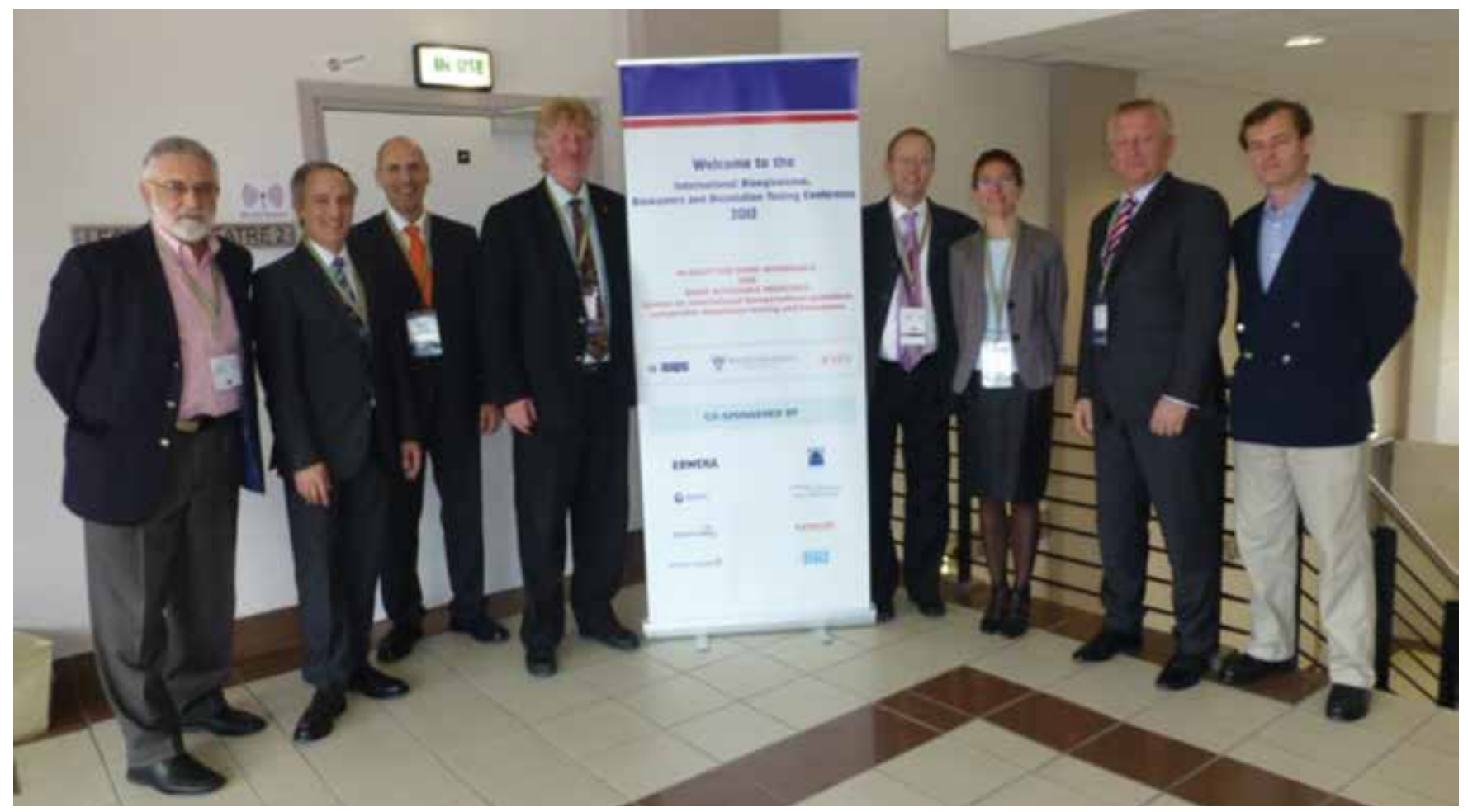

Workshop speakers and organizers, from left to right: Isadore Kanfer, Murray Ducharme, Johannes Krämer, Rod Walker, Raimar Löbenberg, Erika Stippler, Hans Knitter, and Mike Aeroboe. 